\title{
LEVANTAMENTO QUANTITATIVO DE NÓS-DE-PINHO EM UM REMANESCENTE DE FLORESTA OMBRÓFILA MISTA - GENERAL CARNEIRO - PR
}

\author{
Quantitative Survey of Pine Knots in an Araucaria Forest \\ Fragment - General Carneiro, Parana State, Brazil
}

\author{
Mariah Juliane Pereira Vargas ${ }^{1}$ \\ Carlos Roberto Sanquetta ${ }^{2}$ \\ Karla Simone Weber ${ }^{3}$ \\ Kleber dos Santos ${ }^{4}$
}

\section{Resumo}

O presente estudo foi realizado com o intento de reunir informações de quantificação e localização de nósde-pinho em um remanescente da Floresta Ombrófila Mista. Duas áreas de remanescentes localizadas no município de General Carneiro - PR foram usadas para um levantamento da erva-mate e demais produtos florestais não-madeireiros, dentre eles o nó-de-pinho, sendo denominadas como fazenda Santa Cândida e fazenda Lageado Grande, onde foram instaladas parcelas em conglomerados com 4 subunidades de $25 \mathrm{~m}$ x $10 \mathrm{~m}\left(250 \mathrm{~m}^{2}\right)$ cada, totalizando $1.000 \mathrm{~m}^{2}$, sendo 40 conglomerados na primeira fazenda e 20 na segunda. O processo de amostragem foi o aleatório com pré-estratificação da área, definidos de acordo com o estágio sucessional da floresta (inicial, médio e avançado). O número médio de nós constatado na fazenda Santa Cândida foi de 559,25 unidades/ha, enquanto na Lageado Grande quantificou-se 900,50 unidades/ha, correspondendo a $411,28 \mathrm{~kg} / \mathrm{ha}$ e $617,80 \mathrm{~kg} / \mathrm{ha}$, respectivamente, sendo que as maiores quantidades de nós foram verificadas nos estágios médio e avançado de sucessão. Os pesos unitários médios dos nós foram de $0,74 \mathrm{~kg} /$ unidade e 0,69 kg/unidade nas fazendas Santa Cândida e Lageado Grande, respectivamente. O processo de inventário demonstrou-se insatisfatório quanto a intensidade de unidades amostrais para o nóde-pinho, reflexo da quantidade de parcelas sem incidência de nós. Visto que a análise espacial evidenciou que a presença do nó segue um padrão de alta concentração, ou seja, quando há ocorrência dele é de forma abundante, conclui-se que um inventário conjunto de não-madeireiros não atende as expectativas estatísticas para o nó-de-pinho, explicado pelas características de sua distribuição espacial.

Palavras-chave: Floresta de araucária; Nó-de-pinho; Produto florestal não-madeireiro.

1 Acadêmica do Curso de Engenharia Florestal, UFPR, mariah@ufpr.br

2 Eng. Florestal, M.Sc. Ph.D., Professor do Departamento de Ciências Florestais, UFPR, sanquetta@ufpr.br

3 Eng. ${ }^{a}$ Florestal, Pós-Graduação em Engenharia Florestal, UFPR, karlasimone@gmail.com

4 Eng. Florestal, Pós-Graduação em Engenharia Florestal, UFPR, kleberdsan@yahoo.com.br 


\section{Abstract}

The present study was carried out with the intention to congregate information of quantification and localization of the pine knots in a fragment of Mixed Araucaria Forest. Two fragment areas localized in General Carneiro Town - PR had been used for a survey of erva-mate and other types of non-forest products, including the pine knot, being called as Santa Candida and Lageado Grande farms, where it had been installed cluster plots with 4 subunits of $10 \mathrm{~m} \times 25 \mathrm{~m}\left(250 \mathrm{~m}^{2}\right)$ each, totalizing $1,000 \mathrm{~m}^{2}$, being 40 clusters in the first farm and 20 in the second one. It was used a randon sampling with pre-stratification of the area, defined according to the forest sucessional stage (initial, middle and advanced). The average number of knots found in the Santa Candida farm was 559.25 units/ha, while in the Lageado Grande Farm was 900.50 units/ha, corresponding to $411.28 \mathrm{~kg} / \mathrm{ha}$ and $617.80 \mathrm{~kg} / \mathrm{ha}$, respectively, being that the highest amount of knots that had been verified in middle and advanced stages of succession. The average unitary knot weight was $0.74 \mathrm{~kg} / \mathrm{unit}$ and $0.69 \mathrm{~kg} /$ unit in the Santa Cândida and Lageado Grande farms, respectively. The conducted inventory was unsatisfactory with respect to the sampling intensity for pine knots, as consequence of the amount of zero plots. The spatial distribution of the knots occurs in a concentrated pattern, in accordance with the cluster analysis made, therefore it is concluded that a joint not-lumber inventory does not attend the statistical expectations for the pine knot evaluation.

Keywords: Araucaria forest; Pine knot; Non-timber forest products.

\section{Introdução}

A Floresta Ombrófila Mista, também conhecida como Floresta de Araucária, caracterizase pela presença de Araucaria angustifolia (Bert.) Kuntze. Essa ecorregião limita-se a locais de clima pluvial subtropical, ocorrendo abaixo do Trópico de Capricórnio, em altitudes que vão de 500 a 1.200 metros (CASTELLA; BRIETZ, 2004).

Até o início do século XX, a araucária dominava boa parte das paisagens do Planalto Meridional. Sua área de ocorrência natural no Brasil abrangia uma extensão de quase $200 \mathrm{mil} \mathrm{km²}$. No Paraná cobria 40\% da superfície, em Santa Catarina ocupou 30\% e no Rio Grande do Sul atingiu 25\% do território (KOCH; CÔRREA, 2002). A Floresta de Araucária ocupa ainda pequenas manchas no Estado de São Paulo, Minas Gerais e Rio de Janeiro (REITZ; KLEIN; REIS, 1983). De acordo Reitz e Klein, (1966), no estado do Paraná os pinhais distribuem-se no primeiro, segundo e na parte leste do terceiro planalto, perfazendo uma área original de $100.000 \mathrm{~km}^{2}$. No entanto, Maack (1968) estabeleceu uma cobertura menor da floresta original para o estado, de 73,8 mil $\mathrm{km}^{2}$.

É inegável que a paisagem natural criada pela natureza foi fortemente alterada e que a diversidade de espécies que existia há um século atrás foi perdida durante a devastação dos pinheirais. No Paraná, os remanescentes da Floresta de Araucária compõe cerca de 2,7 milhões de hectares, ou seja, 23\% da área original. Contudo, a maior parte desse remanescente constitui-se de fragmentos bastante alterados em sua composição e estrutura e extremamente desconexos entre si (SANQUETTA, 2005). Atualmente a Floresta de Araucária em estágio avançado de sucessão soma menos de 1\% de sua área original no estado (FUPEF-CNPQ, 2001).

O manejo florestal é um elemento decisivo para perpetuar a sobrevivência da Araucaria angustifolia, uma vez que pode colaborar para estimular a regeneração natural, aumentando também as taxas de crescimento das árvores remanescentes e diminuindo as taxas de mortalidade natural na floresta (SANQUETTA, 2005).

Torna-se cada vez mais evidente que as florestas proporcionam uma gama enorme de produtos e benefícios, muitos dos quais longamente conhecidos e utilizados pela comunidade rural e pelos habitantes tradicionais e, em alguns casos, ainda essenciais à sua sobrevivência.

No entanto, o manejo madeireiro nos remanescentes da Floresta Ombrófila Mista está praticamente paralisado em virtude da Resolução CONAMA 278/01, a qual dispõe sobre a suspensão de autorizações concedidas de corte e exploração de espécies ameaçadas de extinção na Floresta Ombrófila Mista. Por isso, formas alternativas de utilizar racionalmente os recursos deste bioma devem ser pesquisadas. Uma alternativa são os produtos florestais não-madeireiros, dentre eles o nó-de-pinho. 
Após a morte e decomposição do pinheiro, restam os nós-de-pinho no solo, os quais possuem em média $30 \mathrm{~cm}$ de comprimento, podendo variar conforme o tamanho do pinheiro. A sua forma é ligeiramente cônica, muitas vezes contendo pequenos sulcos paralelos em sua extensão, proporcionando uma superfície pouco lisa. É de notável resistência, podendo permanecer intacto durante vários anos (TORTATO, 1998). O nó-de-pinho se forma no local de inserção dos galhos como um núcleo nodoso de elevado peso específico e com alto poder calorífico, muito utilizado como fonte de energia e também em peças artesanais em virtude de sua coloração e formas atraentes (INOUE; RODERJAN; KUNIYOSHI, 1984).

Dados do IBGE (2003) apontam que em 2003, no Brasil, o total coletado de nós-de-pinho foi de 26.485 toneladas, o que representa, em termos monetários, a quantia de 551 mil reais, sendo que $93,58 \%$ dos nós-de-pinho são provenientes do estado do Paraná, o restante fica distribuído entre Santa Catarina (5,04\%) e Rio Grande do Sul (1,38\%).

Não se encontram na literatura informações específicas sobre quantificação, localização e espacialização da ocorrência do nó-de-pinho em remanescentes da Floresta de Araucária, razão pela qual este trabalho pretende fornecer informações sobre a ocorrência do nó-de-pinho nas várias fases sucessionais da Floresta Ombrófila Mista, bem como testar estatisticamente a consistência dos dados de nó-de-pinho quando realizado conjuntamente com o levantamento de outros produtos florestais não-madeireiros como plantas medicinais, espécies comestiveis e ornamentais.

\section{Material e Métodos}

\section{Descrição da área de estudo}

A base de dados deste estudo é procedente das Fazendas Lageado Grande e Santa Cândida, propriedades das Indústrias Pedro N. Pizzato Ltda. Ambas estão situadas no município de General Carneiro, extremo Sul do PR, a cerca de 280 $\mathrm{km}$ de Curitiba. O acesso à área se dá pelas rodovias BR-153 e PR-170. As coordenadas geográficas de referência são: $26^{\circ} 20^{\prime} 35^{\prime \prime}$ e $26^{\circ} 26^{\prime} 13^{\prime \prime}$ s, e $51^{\circ} 19^{\prime} 49^{\prime \prime}$ e $51^{\circ} 25^{\prime} 29^{\prime \prime}$ W.

A fazenda Lageado Grande possui uma área total de 2.187,7 ha, sendo que destes, 546,9 ha são constituídos de florestas em estágio inicial, 328,2 ha em estágio médio e 1.312,6 ha em estágio avançado de sucessão. Para a fazenda Santa Cândida, que possui uma área total de 3.404, 0 ha, o estágio inicial encobre 340,4 ha, o estágio médio 2.042,4 ha e o estágio avançado 1.021,2 ha.

O clima da região, segundo a classificação de Köeppen, é do tipo Cfb (MAACK, 1968). A média do mês mais quente é inferior a $22^{\circ} \mathrm{C}$ e a do mês mais frio é inferior a $18^{\circ} \mathrm{C}$, sem estação seca definida, verão é brando, com geadas severas e freqüentes (IAPAR, 1994). Segundo a Embrapa (1984), a área em estudo é composta de três tipos de solos: Cambissolo Álico, Associação solos Litólicos Álicos + Terra Rocha Estruturada Álica, Associação Terra Bruna Estruturada Álica + Cambissolo Álico. De acordo com a classificação do IBGE (1992), a formação florestal original da área de estudo é classificada como Floresta Ombrófila Mista e encontra-se nos mais diversos estágios sucessionais, que devido às interferências antrópicas resultou em uma floresta com vegetação secundária. A Resolução CONAMA 4/94 conceitua esse tipo de vegetação como sendo resultante de processos naturais de sucessão, após supressão total ou parcial da vegetação primária por ações antrópicas ou causas naturais. As intervenções mais evidentes são a extração seletiva de madeira e lenha, coleta de sementes de valor alimentício, caça e pesca predatórias, manejo com roçadas para condução da erva-mate e a presença, em lugar restrito, de animais domésticos, que agora já estão sendo controladas na área de estudo (SEELIGER; CORDAZZO; BARBOSA, 2002). Observou-se também a grande profusão da taquara dos gêneros Merostachys spp e Chusquea spp ocupando intensamente espaços no local de estudo.

\section{Metodologia}

Os dados utilizados neste trabalho foram obtidos em conjunto do inventário para a quantificação da erva-mate e demais produtos florestais não-madeireiros, dentre eles, o nó-de-pinho. O processo de amostragem adotado foi o aleatório com pré-estratificação da área para alocação das unidades amostrais, utilizando-se parcelas em conglomerados, no formato cruz de malta. As áreas foram estratificadas em três tipos vegetacionais em cartas-imagens confecionadas com imagens do 
satélite Ikonos, seguindo as definições da Resolução 02/94 do CONAMA, que dita a categorização mínima de enquadramento das tipologias de acordo com a fisionomia da floresta nos estágios de sucessão em inicial, médio ou avançado.

As unidades amostrais foram georreferenciadas a partir de seu centro, dado que foram tomadas suas coordenadas em campo, mediante o uso de GPS de navegação. Os conglomerados possuem formato de cruz de malta, com 4 subunidades de $25 \mathrm{~m}$ x $10 \mathrm{~m}$, totalizando $250 \mathrm{~m}^{2}$, e que no cômputo das quatro subunidades resulta em $1.000 \mathrm{~m}^{2}$. A intensidade de amostragem foi de 40 conglomerados na Fazenda Santa Cândida, totalizando 160 subunidades e de 20 conglomerados na Fazenda Lageado Grande, totalizando 80 subunidades. A partir do ponto central do conglomerado foram abertas quatro picadas com $10 \mathrm{~m}$ de comprimento cada, nas direções norte, sul, leste e oeste, onde foram instaladas as subunidades em suas extremidades, conforme ilustra a Figura 1.

FIGURA 1 - Disposição das subunidades no conglomerado.

Figure 1 - Subplots arrangement in the conglomerate.

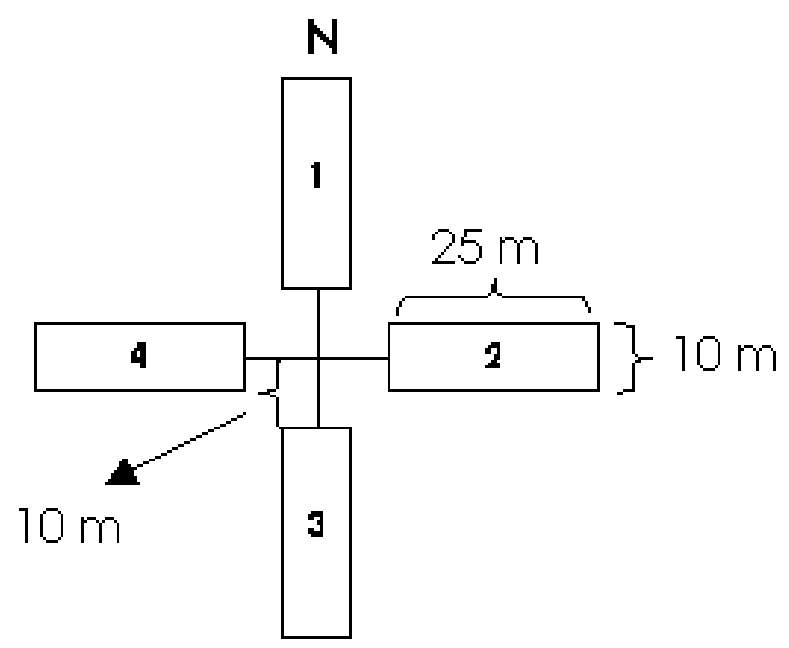

Coleta de dados

O procedimento de localização dos nósde-pinho nas unidades amostrais foi realizado por observação, procurando por nós expostos na superfície, características do terreno, onde, por ex- periência de locais, constataram-se leves afundamentos no relevo, lembrando um aspecto de uma pequena vala, sugerindo uma possível queda de árvore de grande porte que teve seu tronco já apodrecido. Seguindo esta "vala", caso seja de uma antiga araucária, acha-se nó-de-pinho. Também foram utilizadas enxadas, foices e facões que, por serem de metal, no momento em que se bate em um nó-de-pinho faz um barulho característico, o qual indica a sua presença.

Após a localização dos nós-de-pinho dentro das parcelas, foi efetuada a contagem e pesagem na totalidade por subunidade, obtendo-se, assim, um controle de informações sobre as quantidades, pesos totais e médios por subunidade, estágio sucessional da floresta e por fazenda. Esses dados obtidos nas unidades amostrais de cada uma das fazendas permitiram a realização de diversas estimativas e análises do levantamento.

\section{Resultados e Discussão}

A partir da contagem e pesagem dos nós realizada nas subunidades do levantamento, estimou-se a quantidades e pesos médios de nós por hectares para cada uma das fazendas. A Figura 2 compara os valores encontrados para as quantidades médias de nós, sendo que na fazenda Lageado Grande constatou-se 900,5 unidade/ha, valor maior que o constatado para a fazenda Santa Cândida, a qual apresentou 559,3 unidades/ha.

\section{FIGURA 2 - Quantidade média de nós-de-pinho por hectare.}

Figure 2 - Average of pine knots quantity for bectare.

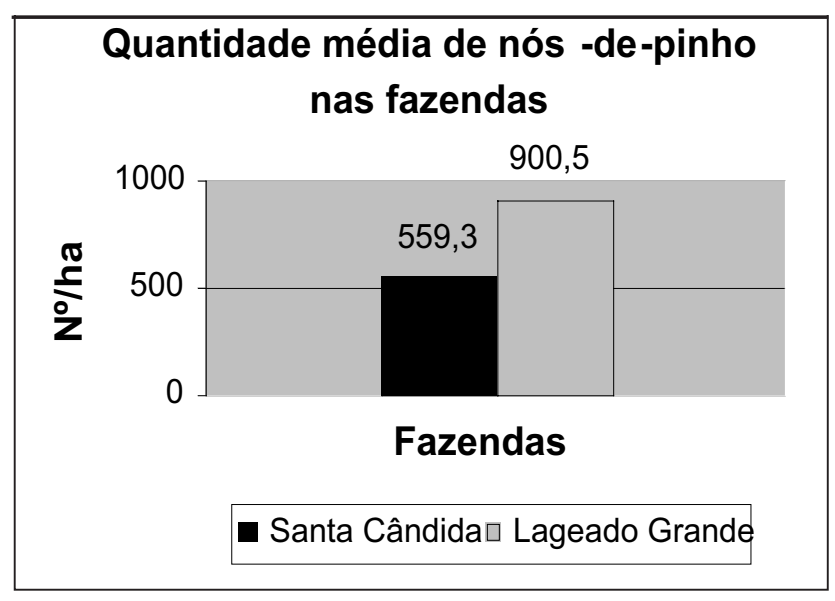


O valor estimado para a média dos pesos nas fazendas estão ilustradas na Figura 3. A fazenda Lageado Grande atingiu 617,8 kg/ha e a Santa Cândida alcançou 411,3 kg/ha.

\section{FIGURA 3 - Peso médio de nós-de-pinho por hectare.}

Figure 3 - Average of pine knots weight for hectare.

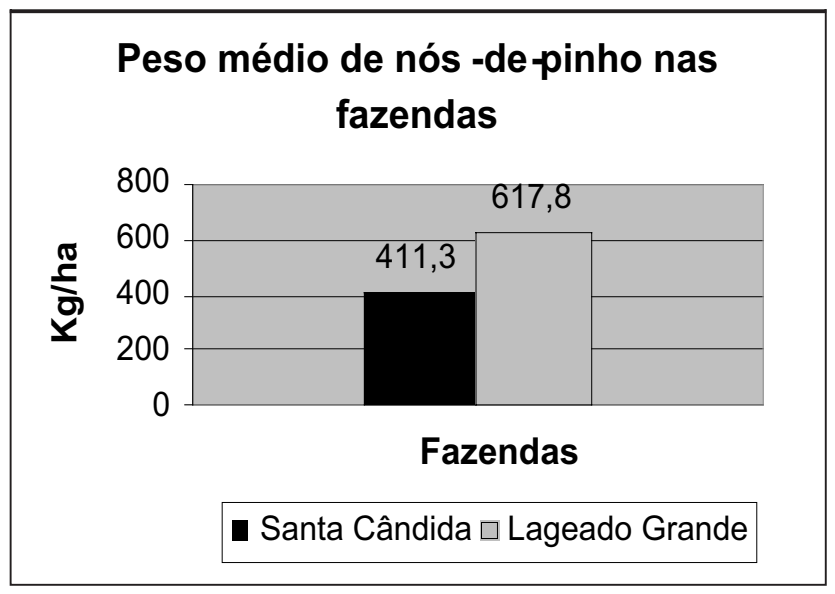

A Figura 4 comprova que para ambas as fazendas a relação entre o número de nós e o peso de nós resultou em valores semelhantes entre as fazendas. O valor alcançado para a Lageado Grande foi de $0,69 \mathrm{~kg} /$ unidade e para Santa Cândida de $0,74 \mathrm{~kg} /$ unidade.

FIGURA 4 - Peso médio unitário de nós-depinho.

Figure 4 - Average of unitary pine knots weight.

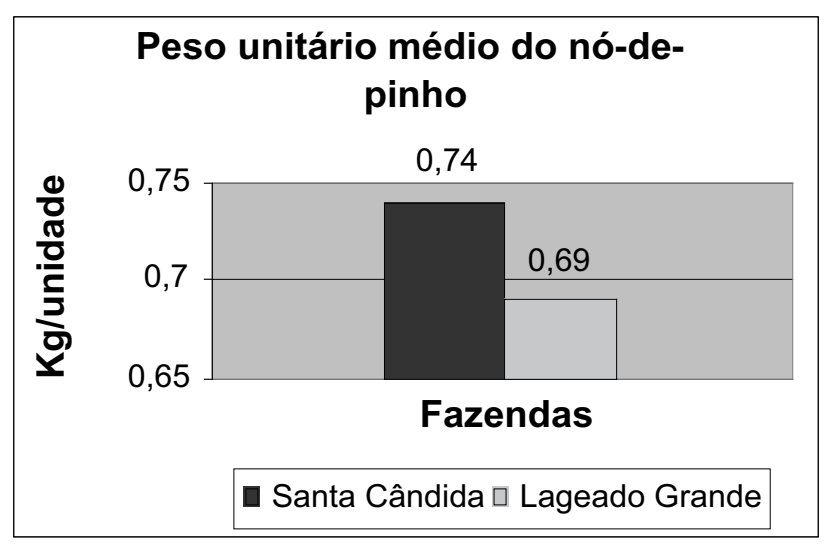

A seguir são apresentados a quantidade e o peso dos nós por fase sucessional e por fazenda. A Figura 5 compara a quantidade de nós en- contrados nas fazendas por fase sucessional. Podese observar que para as duas fazendas em questão, no estágio inicial, foi onde se verificou menos nós. Na fazenda Lageado Grande foram encontradas 368,0 unidade/ha e na fazenda Santa Cândida 52,5 unidades/ha.

FIGURA 5 - Comparação das quantidades de nós por hectare encontrados nos estágios sucessionais entre as fazendas.

Figure 5 - Comparison of pine knots quantity per bectare found in diferent sucessional stages between the study areas.

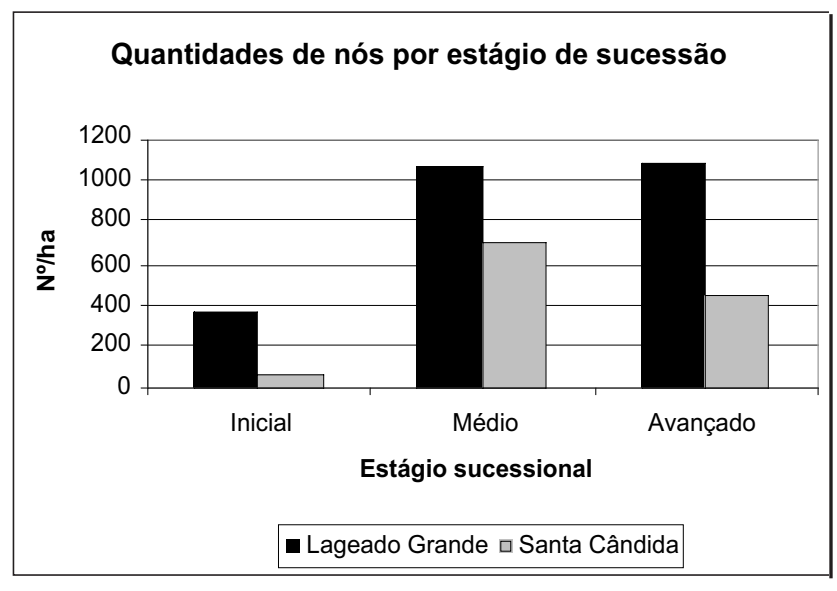

Os estágios médio e avançado de sucessão foram os que apresentaram maiores quantidades de nós, sendo que a fazenda Lageado Grande superou em ambos, com 1.063,3 unidade/ha e 1.081,7 unidade/ha, respectivamente, em detrimento da fazenda Santa Cândida, com 703,8 unidades/ ha e 439,2 unidades/ha, respectivamente.

Para efeitos de comparacão de pesos de nós entre as fazendas também foi gerado um gráfico por fases sucessionais (FIGURA 6). Seguindo aproximadamente o mesmo padrão do gráfico anterior, o estágio inicial foi o que menos contribuiu em peso para ambas as fazendas. A fazenda Santa Cândida apresentou $21,5 \mathrm{~kg} /$ ha, enquanto que na Lageado Grande foram encontrados $222,8 \mathrm{~kg} / \mathrm{ha}$. A fazenda Lageado Grande, da mesma forma que o gráfico anterior, obteve maiores valores para os estágios médio e avançado de sucessão, com 1.025,3 kg/ha e 680,5 $\mathrm{kg} / \mathrm{ha}$ respectivamente. Já a fazenda Santa Cândida apresentou 488,0 kg/ha para o médio e 387,8 $\mathrm{kg} / \mathrm{ha}$ para o médio. 
FIGURA 6 - Comparação dos pesos de nós por hectare encontrados nos estágios sucessionais entre as fazendas.

Figure 6 - Comparison of pine knots weight for bectare found at the different sucessional stages in the study area.

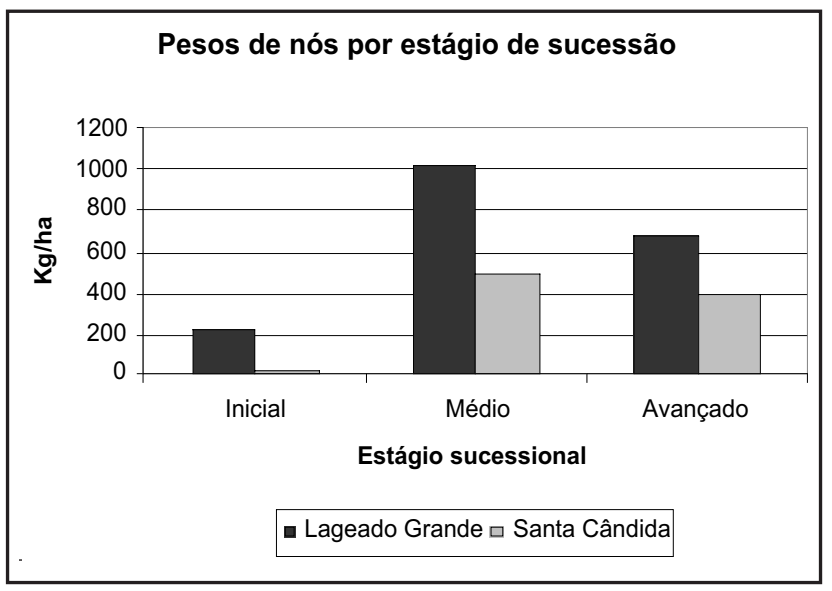

A Tabela 1 apresenta a porcentagem de subunidades nas quais foram encontrados nós-depinhos por estágio sucessional para cada uma das fazendas. Na Fazenda Santa Cândida, o estágio médio foi o que apresentou maior porcentagem, com $66,7 \%$, seguido pelo avançado, com $28,6 \%$ e por fim pelo inicial, com 4,8\%. Já na Fazenda Lageado Grande o estágio sucessional com maior porcentagem de ocorrência de nó-de-pinho também foi o avançado com $61,5 \%$, seguido pelo médio com $23,5 \%$ e permanecendo por último o inicial com $15,4 \%$.

TABELA 1 - Porcentagem de subunidades por estágio sucessional onde foram encontrados nós-depinho.

Table 1 - Percentage of subplots by sucessional stages where pine knots were found.

\begin{tabular}{|c|c|c|c|c|}
\hline \multirow[b]{2}{*}{ Estágio sucessional } & \multicolumn{2}{|c|}{ Santa Cândida } & \multicolumn{2}{|c|}{ Lageado Grande } \\
\hline & Total & $\%$ & Total & $\%$ \\
\hline Inicial & 1,0 & 4,8 & 2,0 & 15,4 \\
\hline Médio & 14,0 & 66,7 & 3,0 & 23,1 \\
\hline Avançado & 6,0 & 28,6 & 8,0 & 61,5 \\
\hline Total & 21,0 & 100,0 & 13,0 & 100,0 \\
\hline
\end{tabular}

Nas Tabelas 2 e 3 estão apresentados o percentual de concentração dos nós em função de sua quantidade encontrada por subunidade. Foi realizada uma divisão por classes de quantidade de nós encontrados por parcela e pelos estágios de sucessão da floresta, onde tem-se que a fazenda Santa Cândida apresenta um maior número de subunidades $(38,1 \%)$ que se encontrou na classe de 1 a 30 nós-de-pinho, padrão de concentração semelhante ao da Fazenda Lageado Grande, em que $38,5 \%$ das subunidades se enquadraram na mesma classe de quantidades de nós.

Considerando que as classes acima de 61 nós por subunidade possam ser qualificadas como de alta concentração de nós-de-pinho, têm-se para as Fazendas Santa Cândida e Lageado Grande, res- pectivamente, 42,9\% e 61,5\% das subunidadess com valores enquadrados nesta condição. A alta concentração de nós no terreno está diretamente relacionada ao tombo da copa da araucária, pois esses são a inserção dos galhos no tronco das árvores que uma vez estiveram presentes no local, estabeleceram-se no chão e sofreram a ação do tempo, dando início à degeneração do material lenhoso, restando, assim, o nó-de-pinho depositado no solo. Nas subunidades que tiveram menos de 61 nós-de-pinho, inclusive os de nenhuma ocorrência, a decomposição de parte dos nós de uma copada, devido ao grande tempo exposto no solo e ao carregamento dos nós para outros locais decorridos da ação da chuva, explica o fato de serem encontrados nós-de-pinho isolados, em pe- 
quena quantidade ou a ausência deles nas subunidades. Alguns fatores foram limitantes na localização dos nós no solo, são eles: a alta pedregosidade, que dificulta a abertura de buracos no solo para a procura dos nós; e a alta concentração de taquarais nas duas áreas de pesquisa, sendo este último agravado pelo manto entrelaçado das taquaras que formam uma camada superficial de 0,6 $\mathrm{m}$ a $1,8 \mathrm{~m}$ de altura, dificultando a visualização dos nós.

TABELA 2 - Distribuição relativa (\%) das subunidades em função de classes de concentração de nós-de-pinho na fazenda Santa Cândida.

Table 2 - Relative distribution (\%) of the subunities in function of concentration classes of pine knots in the Santa Candida farm.

\begin{tabular}{|c|c|c|c|c|c|c|}
\hline \multicolumn{7}{|c|}{ SANTA CÂNDIDA } \\
\hline \multirow[b]{2}{*}{ Estágio sucessional } & \multirow{2}{*}{$\begin{array}{l}\text { N. }{ }^{\circ} \text { de } \\
\text { subunidades } \\
\text { Total absoluto }\end{array}$} & \multicolumn{5}{|c|}{$\begin{array}{l}\text { Porcentagem de subunidades por classe de } \\
\text { quantidade de nó-de-pinho }\end{array}$} \\
\hline & & 1 a 30 & 31 a 60 & 61 a 90 & 91 a 150 & $>150$ \\
\hline Inicial & 1,0 & 100,0 & 0,0 & 0,0 & 0,0 & 0,0 \\
\hline Médio & 14,0 & 35,7 & 28,6 & 0,0 & 14,3 & 21,4 \\
\hline Avançado & 6,0 & 33,3 & 0,0 & 16,7 & 50,0 & 0,0 \\
\hline Todos os estágios & 21,0 & 38,1 & 19,0 & 4,8 & 23,8 & 14,3 \\
\hline
\end{tabular}

TABELA 3 - Distribuição relativa (\%) das subunidades em função de classes de concentração de nós-de-pinho na fazenda Lageado Grande.

Table 3 - Relative distribution (\%) of the subunities in function of concentration classes of pine knots in the Lageado Grande farm.

\begin{tabular}{|c|c|c|c|c|c|c|c|}
\hline \multirow[b]{3}{*}{ Estágio sucessional } & & \multicolumn{6}{|c|}{ LAGEADO GRANDE } \\
\hline & $\begin{array}{l}\text { N. }{ }^{\circ} \text { de } \\
\text { subunidades }\end{array}$ & \multicolumn{6}{|c|}{$\begin{array}{l}\text { Porcentagem de subunidades por classe de } \\
\text { quantidade de nó-de-pinho }\end{array}$} \\
\hline & Total absoluto & 1 a 30 & 31 a 60 & 61 a 90 & $91 \mathrm{a}$ & & $>150$ \\
\hline Inicial & 2,0 & 50,0 & 0,0 & 0,0 & 0,0 & 50,0 & \\
\hline Médio & 3,0 & 33,3 & 0,0 & 0,0 & 33,3 & 33,3 & \\
\hline Avançado & 8,0 & 37,5 & 0,0 & 0,0 & 25,0 & 37,5 & \\
\hline Todos os estágios & 13,0 & 38,5 & $\mathbf{0 , 0}$ & 0,0 & 23,1 & 38,5 & \\
\hline
\end{tabular}

Conforme já mencionado anteriormente, os dados analisados foram obtidos considerando um conjunto de produtos florestais não-madeireiros, o que implicou em resultados não específicos para o nó. Os resultados da análise estatística evidenciaram variâncias elevadas para as duas fazendas e nos estágios sucessionais da Floresta de Araucária. Considerando um erro de amostragem máximo de $20 \%$ com $90 \%$ de grau de confiabilidade, o número de unidades amostrais mostrou-se insuficiente para tomarmos os resultados levantados como provindos de um inventário definitivo para o nó-de-pinho. 


\section{Conclusões}

Com base nas informações obtidas de quantificação e espacialização dos nós-de-pinho para as duas fazendas em questão, é possível concluir que:

- As maiores quantidades de nós-de-pinho foram verificadas no estágio médio de sucessão para a fazenda Santa Cândida e no estágio avançado para a fazenda Lageado Grande. Em virtude disso, recomenda-se que, para fins de exploração do produto florestal não-madeireiro, as unidades amostrais estejam mais concentradas em locais de estágio médio e avançado de sucessão.

- Em uma amostragem não tendenciosa, seja ela aleatória ou sistemática, haverá grande incidência de parcelas que não apresentarão a ocorrência de nó-de-pinho, pois a distribuição espacial mostrouse heterogênea.

- Devido à queda da copa, a distribuição espacial dos nós-de-pinho demonstrou ser agrupada.

- Observou-se adensamento abundante de taquara em algumas parcelas da região de estudo, o que dificultou a visualização e coleta dos nós. Para trabalhos subseqüentes, em locais onde a entrada for inviável para fins de exploração ou pesquisa, recomenda-se excluí-las da população onde a amostragem ou coleta for realizada.

- Pode-se considerar que o levantamento foi piloto para o nó-de-pinho, pois foi realizado em conjunto com outros produtos florestais nãomadeireiros. Em decorrência disso, sugere-se para trabalhos posteriores que a intensidade das unidades amostrais e a sua alocação seja realizada especificamente para o nó-de-pinho.

- Não há informações na literatura a respeito da quantificação e localização dos nós-de-pinho em remanescentes florestais, razão pela qual outros trabalhos de pesquisa sobre o tema devem ser encorajados.

\section{Referências}

BOITEAUX, H. Madeiras de construção de Santa Catarina. Florianópolis: IBGE, 1947. (IBGE. Publicação, 27).

CASTELlA, P. R.; BRITEZ, R. M. A Floresta com Araucaria no Paraná. Brasília:[S. n.], 2004.

EMBRAPA (Empresa Brasileira de Pesquisa Agropecuária). Levantamento de reconhecimento dos solos do Estado do Paraná. Boletim de Pesquisa, Londrina, v. 27, n. 1- 2, 1984.
FUPEF-CNPq (Fundação de Pesquisas Florestais do Paraná- Conselho Nacional de Desenvolvimento Científico e Tecnológico . Conservação do bioma floresta com Araucária. Curitiba: Relatório Final, v. 1 e 2, p. 456. 2001.

IAPAR (Instituto Agronômico do Paraná). Cartas climáticas básicas do estado do Paraná. Londrina: IAPAR, 1994. p. 49. (Documentos; 18).

IBGE (Instituto Brasileiro de Geografia e Estatística). Produção da extração vegetal e da Silvicultura. Brasil: IBGE, v 18, p. 41. 2003.

IBGE. Manual técnico da vegetação brasileira. Rio de janeiro, RJ: IBGE, 1992.

INOUE, M. T.; RODERJAN, C. V.; KUNIYOSHI, S. Y. Projeto Madeira do Paraná. Curitiba: Fupef, 1984.

KOCH, Z.; CORRÊA, M. C. Araucária, a floresta do Brasil Meridional. Curitiba: Olhar Brasileiro, 2002.

MAACK, R. Geografia física do Estado do Paraná. Curitiba: CODEPAR, 1968.

REITZ, R.; KLEIN, R. M. Araucariaceae. In: REITZ, R. Flora ilustrada Catarinense. Itajaí: ARAU, 1966. p. 62.

REITZ, R.; KLEIN, R.; REIS, A. Projeto Madeira do Rio Grande do Sul. Itajaí, 1983.

RIZZINI, C. T. Plantas do Brasil: árvores e madeiras úteis do Brasil: manual de dendrologia brasileira. 2. ed. São Paulo, SP: Edgard Blücher, 1978.

SANQUETTA, C. R. Perspectivas da recuperação e do manejo sustentável das florestas de araucária. ComCiência, ago. 2005. Disponível em: <http://www.comciencia.br/reportagens/2005/08/09.shtml>. Acesso em: jan. 2006.

SEELIGER, U.; CORDAZZO, C.; BARBOSA, F. Os sites e o programa brasileiro de pesquisas ecológicas de longa duração. Belo Horizonte:[S. n.], 2002. v. 1, p 167-181.

TORTATO, M. A. Cultivo de orquídeas em nó de pinho. Boletim da Coordenadoria das Associações Orquidófilas do Brasil, Porto Ferreira, n. 34, p. 118-122, 1998.

Recebido: 09/04/2005 Aprovado: 30/09/2005 